\title{
Regular el juego para prevenir la adicción: hoy más necesario que nunca
}

\section{Regulating gambling to prevent addiction: more necessary now than ever}

\author{
Mariano Chóliz*, Jerónimo Saiz-Ruiz**. \\ "Facultad de Psicología. Universidad de Valencia. Consejo Asesor de Juego Responsable. Dirección General de Ordenación del \\ Juego. **Hospital Ramón y Cajal. Universidad de Alcalá. CIBERSAM. IRYCIS. Consejo Asesor de Juego Responsable. Dirección \\ General de Ordenación de Juego.
}

\section{Resumen}

En mayo de 2013 apareció la quinta edición de la clasificación de los trastornos mentales (DSM-5) de la American Psychiatric Association (APA, 2013), en la cual se incluye el trastorno de juego dentro de la categoría de trastornos adictivos, que es algo que era demandado desde hace décadas desde los ámbitos clínicos, sociales y científicos. El juego de azar, que según la propia APA tiene la misma consideración que las drogas o el alcohol en cuanto a la activación de los circuitos cerebrales de recompensa y a las consecuencias clínicas del trastorno del juego, no tiene, sin embargo, esa consideración desde el principal ámbito desde donde se debe llevar a cabo la prevención de la adicción, que es en los poderes públicos.

El trabajo que presentamos es una propuesta de regulación del juego para llevar a cabo desde la Administración mediante acciones de gobierno que competen a diferentes niveles de la misma (estatal, autonómico e incluso local), con el objetivo final de prevenir la adicción al juego. Dicha propuesta ha sido presentada tanto a la Dirección General de Ordenación del Juego como a la Cámara del Congreso de los Diputados, esta última en el marco de la Comisión Mixta Congreso-Senado para el Estudio del Problema de las Drogas. En este trabajo se defiende la propuesta de regulación desde la evidencia que proporcionan los estudios científicos en materia de prevención de las adicciones.

Palabras clave: Adicción al Juego; Prevención; Regulación del Juego; Trastorno de juego; Juego Responsable.

\begin{abstract}
The American Psychiatric Association published the 5th Edition of DSM in May 2013, in which the gambling disorder is included within the category of addictive disorders -a long-standing and recurrent demand from the clinical, social and scientific fields. Nevertheless, the harmful effects of gambling have not been considered by the Government, which is the main area of addiction prevention.

The present article is a proposal for the regulation of gambling by the Government through the different levels of the State (national, regional and even local), which has the ultimate goal of preventing gambling addiction. This proposal has been presented to the Chamber of Deputies of the Congress, as part of the Congress-Senate Joint Committee for the Study of Drug Problems. The proposed regulation is based on the evidence provided by scientific studies on the prevention of addiction.

Keywords: Gambling Addiction; Prevention; Gambling Regulation; Gambling Disorder; Responsible Gambling.
\end{abstract}




\section{Estado actual de la cuestión}

$\mathrm{E}$ 1 DSM-5 (APA, 2013) ha dado cuenta de una demanda largamente esperada, que es el reconocimiento del juego patológico como un trastorno adictivo, incluyéndolo en la misma categoría diagnóstica que las drogodependencias, el alcoholismo o el tabaquismo (Petry, 2006a; Potenza, 2006). Algo similar puede ocurrir con el caso de la adicción a videojuegos en línea, que es el trastorno que el DSM-5 denomina como Internet Gaming Disorder y que actualmente se incluye en la Sección III del referido manual (Carbonell, 2014).

A pesar de que, en lo que se refiere al juego patológico, no ha habido ningún cambio conceptual relevante desde el DSM-III-R (Chóliz, 2014a), la justificación dada por la APA para definir el trastorno de juego como un trastorno adictivo no puede ser más explícita, ya que "los comportamientos de juego patológico activan los sistemas de recompensa de manera semejante a las drogas de abuso y porque éste produce sintomas conductuales similares a los de los trastornos por consumo de sustancias" (APA, 2013, pg. 481).

Actualmente estamos viviendo en España un momento de auténtica expansión del juego como actividad económica y social (Jiménez-Murcia, Fernández-Aranda, Granero y Menchón, 2014), especialmente desde la aparición y desarrollo de las nuevas modalidades de juego online, lo cual se refleja en las sucesivas memorias de la Dirección General de Ordenación del Juego (DGOJ). En 2014 los españoles gastaron en juegos legales de azar 30.053 millones de euros, lo cual supone aproximadamente un 2,8\% del PIB. De esa cantidad, el 23,40\% (7.032 millones de euros) corresponden a las nuevas modalidades de juego no presencial (DGOJ, 2015).

A pesar de la magnitud del fenómeno y de las implicaciones evidentes que tiene la actividad del juego sobre el juego patológico, los programas de prevención son escasos, parciales y, en muchas ocasiones, carecen de una adecuada evaluación. No obstante, la literatura científica, en lo que se refiere al análisis de las variables que tienen una mayor prevalencia en la aparición de problemas de juego, es ciertamente amplia y objetiva (Williams, West y Simpson, 2012).

\section{Principios básicos de la regulación del juego}

La necesidad de implementar programas de prevención de la adicción al juego por parte de la Administración en España se basa en los siguientes principios:

- El juego es una actividad que puede tener riesgos para la salud ya que, a pesar de que existen diferencias personales en vulnerabilidad (Clark, 2014; Lobo et al., 2014) e incluso factores genéticos de predisposición (Ibañez et al., 2003), tanto las características estructurales del juego (Parke y Griffiths, 2007) como las condiciones en las que se implementa (Williams, West y Simpson,
2012) son factores determinantes del desarrollo del trastorno de juego en la población.

- El trastorno de juego tiene graves consecuencias tanto para el jugador como para la familia; en un porcentaje importante de los casos (aproximadamente un 25\% de los jugadores patológicos) cometen actos ilegales para conseguir dinero con el que poder seguir jugando (Granero et al., 2014).

- Como en cualquier problema de salud, y singularmente en el caso de los trastornos adictivos, la forma más eficaz de abordar el problema es mediante la implementación de programas de prevención (Dickson-Gillespie, Rugle, Rosenthal y Fong, 2008) teniendo en cuenta que:

a. Los programas preventivos deben llevarse a cabo desde diferentes ámbitos, si bien las políticas de juego (las iniciativas de la Administración en materia de regulación del juego) se han demostrado como la forma más eficaz de prevención del juego excesivo (Williams, West y Simpson, 2012).

b. Una vez que se ha desarrollado el trastorno, la persona se ve incapaz de controlar su conducta de juego, en la que predomina la impulsividad (Blanco et al., 2009), incluso a pesar de que esté informado de las consecuencias negativas del juego excesivo. Es necesario, por lo tanto, que exista un adecuado control externo, cuya forma más eficaz son las políticas de juego.

c. La prevención de la adicción al juego es incompatible con la promoción del juego excesivo (Williams, 2014), lo cual hace todavía más necesarias las políticas de regulación por parte de la Administración puesto que el juego, como actividad económica, basa sus beneficios empresariales en el consumo elevado (Chóliz, 2014b). No obstante, en la actualidad nos encontramos con el hecho de que es verdaderamente difícil legislar en materia de juego de forma que se prevengan los trastornos asociados al mismo, lo cual nos recuerda a un problema similar al ocurrido con el alcohol y el alcoholismo (Rodríguez-Martos, 2007)

A pesar de lo dicho hasta ahora, la aparición del trastorno del juego es un problema complejo que también depende de otros factores de vulnerabilidad que hay que tener en cuenta (Clark, 2014), si bien esto es algo que trasciende a este trabajo y corresponde a otras instancias (educativas, sanitarias, etc.) el desarrollar programas de prevención que atiendan a dichas variables (biológicas, psicológicas, etc.).

\section{Propuesta de regulación del juego}

La propuesta de regulación del juego que presentamos se articula en torno a tres ejes, que incluyen las principales variables responsables de la adicción al juego cuyo potencial 


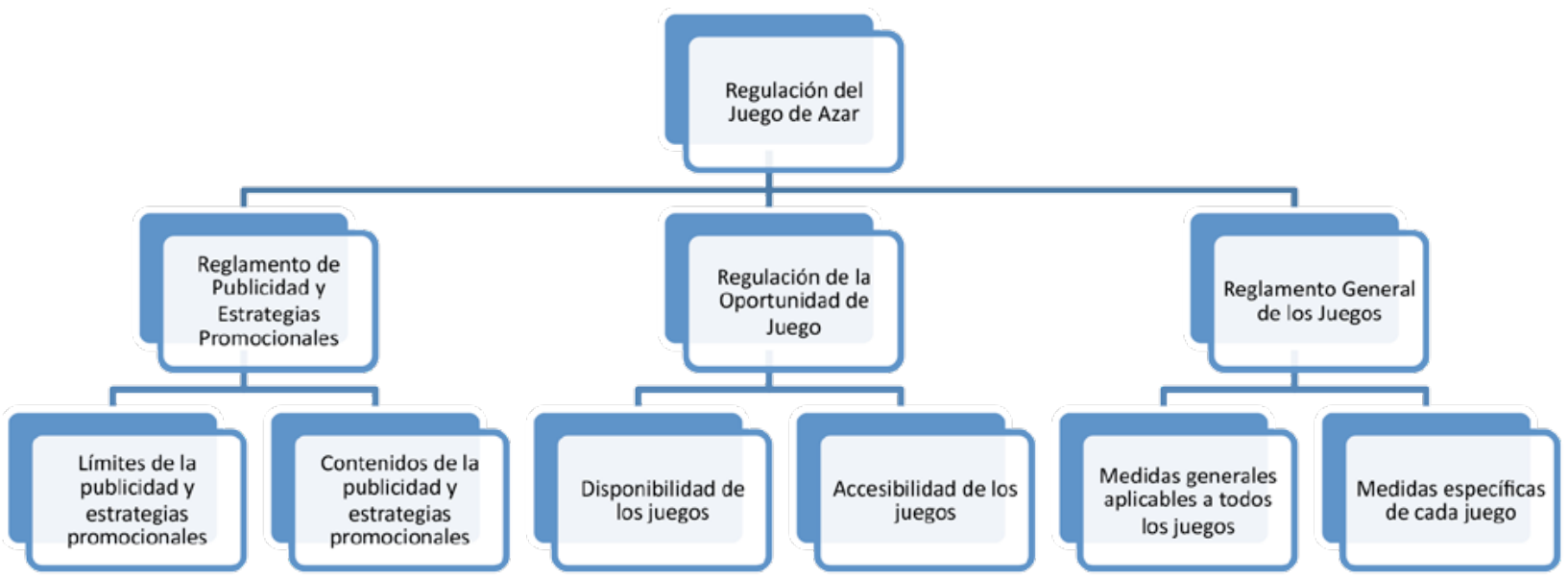

Figura 1. Esquema general del proceso de regulación del juego

adictivo puede reducirse mediante las adecuadas políticas de juego (ver Figura 1):

- Regulación de la publicidad y estrategias promocionales

- Regulación de la oportunidad de jugar, cuyas variables más relevantes son la disponibilidad y accesibilidad del juego, y

- Regulación de las características estructurales de los propios juegos, con el objetivo de disminuir el potencial adictivo de los mismos.

A continuación se desarrollan las diferentes acciones concretas que pueden llevarse a cabo en cada uno de estos ejes:

\section{Publicidad del juego y de las estrategias promocionales}

A pesar de que es preciso establecer regulaciones específicas de la publicidad del juego para prevenir la aparición de trastornos derivados del mismo (Binde, 2014), la aparición del juego online en España ha venido acompañada de un incremento en publicidad y técnicas promocionales, antes incluso de que se este se legalizara y en ausencia de reglamentos específicos de regulación de la publicidad por parte de los sucesivos gobiernos.

Es por ello que es necesario un reglamento de publicidad, que debería girar en torno a dos aspectos esenciales: los límites de la publicidad y los contenidos de la publicidad

Límites de la publicidad. Como estrategia general, la publicidad del juego debería estar limitada a los espacios en los que se lleva a cabo el juego: salones de bingo, casinos, salas de juego y las propias webs de juego en el caso del juego online. En el caso de que no se produjera esta limitación general, deberían tomarse una serie de medidas regulatorias, tales como las siguientes:

- Limitaciones en prensa y medios de comunicación audiovisual. La publicidad debería restringirse en franjas horarias (en la programación de radio y televisión), o secciones (en prensa escrita). De acuerdo con la Ley General de Comunicación Audiovisual, tanto en radio como en televisión e Internet, debería estar restringida al horario de $22 \mathrm{~h}$ a $6 \mathrm{~h}$ del día siguiente.

- Regulación de la publicidad del juego online. En lo que se refiere a la publicidad del juego online a través de Internet, esta deberá tener una regulación específica, ya que las propias características del funcionamiento de Internet pueden hacer que la publicidad no sólo sea intrusiva, sino que se convierta en una técnica de instigar a jugar cuando se abren automáticamente las webs de juego, incluso aunque se estén realizando otras acciones distintas por la Red. Así pues, en Internet, la publicidad del juego online debería realizarse exclusivamente dentro de las páginas web de los juegos, y una vez se haya accedido a las mismas, prohibiendo explícitamente la aparición de banners, vínculos a páginas de juego fuera de dichas webs (como en la prensa electrónica), apertura automática de webs de juego, etc.

Contenidos de la publicidad. El juego es una actividad potencialmente adictiva, que comparte algunas características con el tabaquismo y alcoholismo, pero que también tiene ciertas singularidades, lo cual debería tenerse en cuenta.

- Aspectos regulatorios comunes a otras adicciones. Al igual que ocurre con la publicidad del alcohol, el juego no podrá dirigirse a menores de edad, ni podrá utilizarse la presencia de menores para publicitar el juego; no podrá asociarse el juego a la mejora del éxito profesional, ni dar impresión de que favorece el estado de ánimo positivo, ayuda a resolver conflictos, etc.; no se deberá estimular el juego inmoderado u ofrecer una imagen negativa de no jugar; deberán establecerse normas claras sobre patrocinio u otras medidas indirectas de publicitar el juego, etc.

- Aspectos regulatorios específicos. Una de las principales variables que afectan al potencial adictivo del juego es la forma como se presenta, ya que éste induce sesgos y 
errores cognitivos que, a su vez, van a tener una relevancia fundamental en el desarrollo y mantenimiento de la adicción (Sharpe, 2002). Por ello, ni la publicidad, ni las técnicas promocionales, deberían utilizar estrategias que indujeran a dichos sesgos o errores cognitivos que favorecen el trastorno del juego. Los principales sesgos y técnicas promocionales que habría que eliminar en el caso del juego son: sesgo del experto (inducido por los denominados "bonos de bienvenida"), grandes premios ("big win)" (inducido por los premios acumulados) (Weatherly, Sauter y King, 2004), "casi ganancias", ("near miss") (Habib y Dixon, 2010), etc.

\section{Oportunidad de jugar}

La oportunidad de consumir es una de las variables más relevantes tanto en el desarrollo de la adicción como en las recaídas que se producen en los adictos recuperados (Marlatt y Gordon, 1980, 1985). En el caso del juego, las dos principales variables implicadas son la disponibilidad de los juegos en el entorno y la accesibilidad a los mismos por parte de los jugadores.

Disponibilidad. La disponibilidad se refiere tanto a la oferta de juego, como a la cercanía de los juegos o locales en los que se encuentran. Existe una relación positiva entre disponibilidad del juego y aparición de casos de juego patológico (Welte, Wieczorek, Barnes, Tidwell y Hoffman, 2004), entre los problemas de juego excesivo y patológico con la cercanía de lugares de juego (Pearce, Mason, Hiscock y Day, 2008), con la cantidad de juegos (Grun y McKeigue, 2000) o con la densidad de máquinas de juego electrónico (Storer, Abbott and Stubbs, 2009). Las medidas concretas de regulación en lo que se refiere a la disponibilidad serían las siguientes:

- Tanto el juego presencial como el online deberían llevarse a cabo en salones de juego debidamente acreditados para esta actividad. Ello implicaría la retirada de los bares y restaurantes, tanto las máquinas tipo "B" (coloquialmente conocidas como "tragaperras"), como las más recientes máquinas de apuestas.

- Otorgar las licencias de salones de juego en base a criterios geográficos y de población pero, en cualquier caso, reducir el número de los ya existentes.

Accesibilidad. La accesibilidad hace referencia a la facilidad con la que puede llevarse a cabo el juego. Se considera una de las variables principales en el desarrollo del juego problemático (Thomas, Sullivan y Allen, 2008). Las dimensiones principales que pueden ser objeto de regulación por parte de la Administración harían referencia a los requisitos que se exigen y las condiciones que se establecen para poder jugar, incluso en los juegos ampliamente disponibles. Algunas de las medidas concretas que pueden llevarse a cabo en este sentido son las siguientes:

- Registro General de Interdicciones para cualquier tipo de juego de España (presencial y online) y posibilidad de llevarlo a cabo desde las administraciones del Estado por parte de familiares en primer grado, según el principio de precaución.

- Identificación mediante DNI para cualquier tipo de juego y emisión de factura en el caso de las loterías

- Identificación fehaciente para poder jugar a cualquier juego electrónico y online:

- Incorporación de lector de DNI electrónico en todas las máquinas tipo "B" y en cualquiera otra máquina electrónica de juego o apuestas.

- Habilitación de un sistema de identificación fehaciente en el caso del juego online a través de password obtenido en oficinas de la Administración del Estado, que deberá renovarse periódicamente. Habilitación de un sistema de obtención del pasword para extranjeros a través del consulado de España en los países de origen.

\section{Reglamento General de los Juegos}

A pesar de que son muchas y diversas las características estructurales que están afectando a la adicción al juego, un reglamento debe seleccionar aquellas que son especialmente relevantes y operativizar claramente la forma mediante la cual deben regularse, con el objetivo de reducir el potencial adictivo del juego. En este sentido, los juegos más adictivos son los que tienen una elevada tasa de juego y de reforzamiento (Parke y Griffiths, 2007; Welte, Barnes, Wieczorek, Tidwell y Hoffman, 2007), así como una inmediatez en la respuesta (Chóliz, 2010). Atendiendo a las diferentes modalidades de juego, los juegos electrónicos, o EGM (Electronic Gaming Machines) maximizan estas características y se considera que suelen ser los más adictivos (Brooks, Ellis y Lewis, 2008; Dowling, Smith y Thomas, 2005; Welte et al., 2007), motivo por el cual debe atenderse especialmente su regulación.

Por otra parte, las condiciones bajo las cuales se lleva a cabo el juego también condicionan sobremanera el potencial adictivo de este, potenciando en algunos casos sus efectos (Welte et al., 2004). Se trata de variables como la forma de pago (en metálico o con tarjeta), la ubicación, la posibilidad de consumo de otras sustancias (alcohol, tabaco), etc. Pero sin duda, uno de los factores principales que pueden favorecer el potencial adictivo de los juegos es la utilización de Internet como herramienta en el juego, es decir, el juego online (Petry, 2006b), ya que la Red favorece las principales variables responsables de la adicción: disponibilidad, accesibilidad, inmediatez, rapidez de jugadas, etc. (Griffiths 2003; Griffiths, Parke, Woods y Parke, 2006). De hecho, una de las evidencias más claras es la de que los jugadores online presentan tasas más elevadas de adicción que los jugadores de juegos presenciales (Wood y Williams, 2009; Wood, Williams y Parke, 2012), motivo por el cual la regulación del juego online es uno de los objetivos inmediatos que es preciso llevar a cabo para proteger a personas vulnerables 
(Monaghan, 2009), especialmente en la medida que, hasta la fecha, la legislación se ha visto incapaz de regular adecuadamente el juego online para proteger a jugadores patológicos o jóvenes en riesgo de adicción (Schoen, Hughes, Lewis y Marmon, 2007). De hecho, en un reciente estudio realizado en España, se constata que ha habido un notable incremento del número de jugadores patológicos cuya causa principal de su trastorno es el juego online y, en el caso de los menores de 26 años, el juego online es el principal responsable del trastorno, superando incluso a las máquinas tipo "B" (Chóliz, 2015).

A continuación se exponen algunas de las principales medidas para tratar de reducir los problemas de adicción y juego excesivo.

Medida general: limitación de las pérdidas. Las limitaciones de las pérdidas, también denominadas pre-commitment, han demostrado su eficacia tanto en la prevención de la adicción como en la promoción del juego responsable (Bernhard, Lucas y Jang, 2006; Focal Research Consultants, 2007, 2010; Griffiths, 2012; Omnifacts Bristol Research, 2007; Parke, Rigbye y Parke, 2008; Productivity Commission, 2010; Responsible Gambling Council, 2009; Schrans, Grace and Schellinck, 2004; Williams, 2010). La forma de operativizarlo en un Reglamento General de Juego es impidiendo que una persona pierda más de una determinada cantidad de dinero previamente establecida. Dicha cantidad siempre estará determinada por la Administración y reflejada en el Reglamento General de los Juegos. El control de dichos límites se realiza con una tarjeta inteligente de juego, que se describe a continuación.

Se prevén dos tipos de limitaciones en las pérdidas:

- Pérdidas globales en el juego. Se establecerán límites de pérdidas diarios, semanales y mensuales, que reflejarán la cantidad total de dinero que es posible perder teniendo en cuenta todos los juegos a los que se apueste. Dichos límites serán establecidos por la Administración en el Reglamento General de los Juegos.

- Pérdidas en juegos específicos. Antes del inicio de cualquier juego electrónico, se deberá tener la opción de prefijar con antelación la cantidad máxima que el jugador está dispuesto a perder. Dicha cantidad siempre será inferior a la que la Administración habrá establecido como límite diario de pérdidas en el juego.

La Tarjeta Inteligente de Juego (TIJ) es la herramienta que permitiría llevar a cabo muchas de las técnicas de control de los juegos. Se trataría de una tarjeta inteligente, o smartcard, que recogería la información más relevante acerca del juego, con el objetivo de prevenir un gasto excesivo. Deberá contener, por lo tanto, los datos básicos personales y de juego de su propietario (frecuencia de juego, gasto, pérdidas, etc.). Se trataría de un dispositivo necesario para el funcionamiento de los juegos, acompañada del DNI y será expedida por la Administración.
Sin ánimo de ser exhaustivo, y teniendo en cuenta que tanto las propiedades técnicas como el procedimiento específico de actuación deberían indicarse detalladamente en el Reglamento General de los Juegos, algunas de las características más relevantes que debería tener la TIJ son las siguientes:

- Se trata de una tarjeta personal e intransferible que debe ser utilizada junto con el DNI electrónico en los juegos electrónicos. También se habilitará un sistema de registro en los salones de juego y en otros sectores para los juegos no electrónicos.

- Dicha tarjeta registrará los juegos a los que se ha jugado, el día y la hora, así como el balance de ganancias y pérdidas diario, semanal y mensual.

- Para obtenerla habrá que identificarse fehacientemente (por ejemplo, personarse en dependencias de la Administración Autonómica o Local y acreditarse mediante el DNI). Tendrá una caducidad de 3-6 meses; pasado ese tiempo se deberá volver a activar mediante el procedimiento anterior, con el objetivo de garantizar la identidad del jugador.

- En el Reglamento General de los Juegos se establecerán claramente los límites de pérdidas diarios, semanales y mensuales. En el momento en el que el jugador alcance dichos límites, la TIJ bloqueará la posibilidad de jugar durante el tiempo que se establezca en el Reglamento.

Hay que tener en cuenta que no se trata de una tarjeta de crédito, sino de control del juego. Las máquinas y juegos electrónicos seguirán funcionando con sus dispositivos habituales, que nunca serán tarjetas de crédito o débito, para evitar el gasto excesivo.

Medidas específicas para cada juego de azar. Se trata de normas concretas que están dirigidas a mitigar el efecto que tienen las variables estructurales, y que dependen de cada juego en concreto. Es por ello que se trata de medidas que, a diferencia de las que acabamos de indicar, puede que sean aplicables solamente a un tipo de juego o a unos cuantos de ellos. Se trata de modificaciones técnicas que implican a variables que se ha demostrado científicamente que están influyendo en el desarrollo o mantenimiento de la adicción al juego.

Algunas de las más significativas son las siguientes:

- Dilación de la recompensa en juegos electrónicos, loterías presorteadas, juegos de casino, etc. Se trata de incrementar el tiempo entre: a) la realización de la jugada o apuesta y el resultado (ganancia o pérdida); o b) entre el resultado y el cobro.

- Reducir velocidad de las apuestas. Con esta medida se pretende disminuir la absorción en el juego. Algunas de las estrategias preventivas que impiden esa absorción excesiva son las siguientes:

- Prohibición de las apuestas "en caliente". Las apuestas deben cerrarse antes de iniciarse la competición sobre la que se apuesta. 
- Apuestas sencillas en el caso del juego online. Prohibición de las apuestas combinadas, múltiples, etc. En particular, prohibición de las apuestas cruzadas, por el riesgo de adicción y de seguridad para el jugador (Chóliz, 2013).

- Prohibición de realizar varias partidas de póquer online simultáneamente. Con esta medida también se dificulta que los jugadores profesionales lleven a cabo estrategias en contra de los jugadores neófitos u ocasionales, quienes se encuentran en desventaja, al carecer de las técnicas y herramientas tecnológicas que utilizan los profesionales.

- Limitar las grandes ganancias. Algunas de las medidas a tomar son las siguientes:

- Prohibición de interconexión de máquinas, así como de bingos combinados y otras modalidades que incrementen excesivamente los premios.

- Limitar el porcentaje concedido a premios, especialmente en apuestas y juego online, que en la actualidad es extremadamente elevado en algunos casos y ello provoca patrones de juego excesivo.

\section{Conclusiones}

Las políticas de juego, es decir, los mecanismos regulatorios del Estado sobre la actividad del juego de azar, son la base sobre la que se sustentan el resto de medidas preventivas (Williams, West y Simpson, 2012). Algunas de las dimensiones objeto de regulación, tales como la disponibilidad o accesibilidad, han demostrado su estrecha relación con la aparición de problemas de juego excesivo y de trastornos de juego (Grun y McKeigue, 2000; Pearce et al., 2008; Werte et al., 2004), mientras que otras son probablemente eficaces y habría que demostrar su eficacia una vez implantadas en un sistema de regulación. Estas medidas no sólo resultan necesarias para prevenir la aparición de la adicción al juego, sino también para facilitar el control en quienes ya padecen dicho trastorno. La responsabilidad de desarrollarlas e implementarlas corresponde a los poderes públicos, si bien deben complementarse con medidas educativas, familiares, comunitarias o de los propios servicios de salud.

Se trata de una propuesta que, aunque plausible y con medidas probablemente eficaces, debería estar sometida continuamente a un proceso de evaluación de sus efectos, con el objetivo de ir depurando los procedimientos y técnicas que se plantean. Deberían establecerse criterios objetivos de evaluación, tales como proceder a evaluación de la aparición de trastornos de juego o de demandas de asistencia antes y después de la aplicación de las medidas; seleccionar grupos poblacionales concretos sobre los que observar el efecto de las medidas establecidas; implementación de forma secuencial de algunas de las medidas y observar el efecto que van teniendo en la población general o en ámbitos específicos, etc.
Los objetivos principales de esta propuesta de regulación del juego no son otros que la prevención de la adicción al juego y la reducción del riesgo de quienes ya manifiestan patrones desadaptativos de juego, con el objetivo de compaginar el ejercicio de una actividad lúdica con la preservación del superior derecho a la salud de los ciudadanos.

\section{Referencias}

APA, American Psychiatric Association (2013). Diagnostic and statistical manual of mental disorders (5th Edition). Washington, DC: American Psychiatric Association.

Bernhard, B.J., Lucas, A.F. y Jang, D. (2006). Responsible Gaming Device Research Report. International Gaming institute. University of Nevada, Las Vegas.

Binde, P. (2009). Exploring the impact of gambling advertising: An interview study of problem gamblers. International Journal of Mental Health and Addiction, 7, 541-554. doi.org/10.1007/s11469-008-9186-9

Blanco, C., Potenza, M.N., Kim, S.W., Ibáñez, A., Zaninelli, R., Saiz-Ruiz, J. y Grant, J.E. (2009). A pilot study of impulsivity and compulsivity in pathological gambling. Psychiatry Research, 15(167), 161-168. doi.org/10.1016/j. psychres.2008.04.023

Brooks, G., Ellis, T. y Lewis, C. (2008). Pachinko: A Japanese addiction? International Gambling Studies, 8, 193-205. doi.org/10.1080/14459790802168958

Carbonell, X. (2014). La adicción a los videojuegos en el DSM-5. Adicciones, 26, 91-95.

Chóliz, M. (2010). Experimental analysis of the game in pathological gamblers: effect of the immediacy or the reward in slot machines. Journal of Gambling Studies, 26, 249-256. doi.org/10.1007/s10899-009-9156-6

Chóliz, M. (2013). Informe sobre la legalización de las slot online y las apuestas cruzadas en España. Informe presentado a la Dirección General de Ordenación del Juego (Ministerio de Hacienda y Administraciones Públicas), marzo de 2013.

Chóliz, M. (2015). The challenge of online gambling: the effect of legalization on the increase in online gambling addiction. Journal of Gambling Addiction, junio 2015. doi:10.1007/s10899-015-9558-6

Chóliz, M. (2014a). Historia de una adicción: del vicio al Trastorno del Juego. Revista de Medicina Psicosomática y Psiquiatría de Enlace, 111, 84-88.

Chóliz, M. (2014b). Juego Ético: un compromiso en la prevención de la adicción al juego desde el ámbito político y social. Revista Española de Drogodependencias, 39, 5-9. Recuperado de: www.aesed.com/descargas/revistas/ v39n4_editorial1.pdf

Clark, L. (2014). Disordered gambling: the evolving concept of behavioral addiction. Annals of the New York Academy of Science, 1327, 46-61. doi:10.1111/nyas.12558.

DGOJ, Dirección General de Ordenación del Juego (2015). Memoria Anual 2014. Ministerio de Hacienda. 
Recuperado de la web el 29 de junio de 2015 en http:/ / www.ordenacionjuego.es/es/estudios-informes

Dickson-Gillespie, L., Rugle, L., Rosenthal, R. y Fong, T. (2008). Preventing the Incidence and Harm of Gambling Problems. Journal of Primary Prevention, 29, 37-55. doi.org/10.1007/s10935-008-0126-z

Dowling, N., Smith, D. y Thomas, T. (2005). Electronic gaming machines: Are they the 'crack-cocaine' of gambling? Addiction, 100, 33-45. doi.org/10.1111/j.13600443.2005.00962.x

Focal Research Consultants (2007). Assessment of the behavioural impact of responsible gaming device (RGD) features: Analysis of Nova Scotia player-card data - the Windsor trial. Informe técnico preparado para the Nova Scotia Gaming Corporation. 108

Focal Research Consultants (2010). Evaluating the Impact of the "My-Play" System in Nova Scotia: Phase 1: Regular VL Player Benchmark Survey. October 2010. http:/ /www.nsgamingfoundation.org/uploads/Research/Technical\%20 Report\%20Phase\%201\%20My-Play\%20Benchmark\%20 Final\%20\%20_Focal_\%20Jan\% 2028\%202011.pdf

Granero, R., Penelo, E., Stinchfield, R., Fernández-Aranda, F., Aymamí, N., Gómez-Peña, M., ... Jiménez-Murcia, S. (2014). Contribution of Illegal Acts to Pathological Gambling Diagnosis: DSM-5 Implications. Journal of Addictive Disorders, 33, 41-52. doi: 10.1080/10550887.2014.882730.

Griffiths, M. D. (2003). Internet gambling: Issues, concerns and recommendations. CyberPsychology \& Behavior, 6, 557-568. doi.org/10.1089/109493103322725333

Griffiths, M., Parke, A., Wood, R. y Parke, J. (2006). Internet gambling: An overview of psychosocial impacts. UNLV Gaming Research \& Review Journal, 10, 27-39. doi.org/10.4018/978-1-59140-993-9.ch033

Griffiths, M.D. (2012). Internet gambling, player protection, and social responsibility. En R.J. Williams, R.Wood y J. Parke (eds.), Routledge International Handbook of Internet Gambling (pp. 227-249). Routledge: U.K.

Grun, L. y McKeigue, P. (2000). Prevalence of excessive gambling before and after introduction of a national lottery in the United Kingdom: Another example of the single distribution theory. Addiction, 95, 959-966. doi.org/10.1046/j.1360-0443.2000.95695912.x

Habib, R y Dixon, M.R. (2010). Neurobehavioral evidence for the "near miss" effect in pathological gamblers. Journal of Experimental Analysis of Behavior, 93, 313-328. doi: 10.1901/jeab.2010.93-313.

Ibáñez, A., Blanco, C., Perez de Castro, I., Fernandez-Piqueras, J., y Saiz-Ruiz, J. (2003). Genetics of pathological gambling. Journal of Gambling Studies, 19(1), 11-22.

Jiménez-Murcia, S., Fernández-Aranda, F., Granero, R. y Menchón, J.M. (2014). Gambling in Spain: update on experience, research and policy. Addiction, 109, 15951601. doi: 10.1111/add.
Lobo, D.S., Aleksandrova, L., Knight, J., Casey, D.M., El-Guebaly, N., Nobrega, J.N. y Kennedy, J.L. (2014). Addiction-related genes in gambling disorders: new insights from parallel human and pre-clinical models. Molecular Psychiatry, Sep 30. doi: 10.1038/mp.2014.113.

Marlatt, G.A. y Gordon, J.R. (1980). Determinants of relapse: Implications for the maintenance of behavior change. En: P.O. Davidson y S.M. Davidson (eds), Behavioral Medicine: Changing Health Lifestyles (pp. 410-452). New York: Brunner/Mazel.

Marlatt, G.A., y Gordon, J.R. (1985). Relapse Prevention: Maintenance Strategies in the Treatment of Addictive Behaviors. New York: Guilford Press.

Monaghan, S. (2009). Internet gambling-Not just a fad. International Gambling Studies, 9, 1-4.

Omnifacts Bristol Research. (2007). Nova Scotia player card research project: Stage III research report.

Parke, J. Rigbye, J. y Parke, A. (2008). Cashless and card-based technologies in gambling: A review of the literature. December 2008. Commissioned by the U.K. Gambling Commission.

Parke, J. y Griffiths, M. (2007). The role of structural characteristics in gambling. En G. Smith, D. C. Hodgins y R.J. Williams (Eds.), Research and measurement issues in gambling studies (pp. 217-249). Burlington, MA: Elsevier.

Pearce, J., Mason, K., Hiscock, R. y Day, P. (2008). A national study of neighbourhood access to gambling opportunities and individual gambling behaviour. Journal of Epidemiology and Community Health, 62, 862-868. doi. org/10.1136/jech.2007.068114

Petry, N. M. (2006a). Should the scope of addictive behaviors be broadened to include pathological gambling? Addiction, 101, 152-160. doi.org/10.1111/j.13600443.2006.01593.x

Petry, N. M. (2006b). Internet gambling: An emerging concern in family practice medicine? Family Practice, 23, 421-426. doi.org/10.1093/fampra/cml005

Potenza, M. (2006). Should addictive disorders include non-substance related conditions? Addiction, 101, 142151. doi.org/10.1111/j.1360-0443.2006.01591.x

Productivity Commission. (2010). Gambling Inquiry Report. Canberra: Commonwealth of Australia.

Responsible Gambling Council (2009). Play Information and Management Systems. Responsible Gambling Council, Toronto, Ontario.

Rodríguez-Martos, A. (2007). ¿Por qué es tan difícil legislar sobre alcohol en España?. Adicciones, 19, 325-332.

Schoen, J. E., Hughes, D., Lewis, P. A. y Marmon, R. (2007). Casino City, Inc. V U.S. Department of Justice Campus access to Internet Gambling and the First Amendment. Journal of the International Academy for Case Studies, 13, 6782.

Schrans, T., Grace, J. y Schellinck, T. (2004). 2003 NS VL responsible gaming features evaluation: Final report. Infor- 
me técnico preparado por Focal Research Consultants Ltd. para the Nova Scotia Gaming Corporation, Halifax, Nova Scotia, October 2004.

Sharpe, L. (2002). A reformulated cognitive-behavioral model of problem gambling: A biopsychosocial perspective. Clinical Psychology Review, 22, 1-25. doi.org/10.1016/ S0272-7358(00)00087-8

Storer, J., Abbott, M. y Stubbs, J. (2009). Access or adaptation? A meta-analysis of surveys of problem gambling prevalence in Australia and New Zealand with respect to concentration of electronic gaming machines. International Gambling Studies, 9, 225-244. doi. org/10.1080/14459790903257981

Thomas, A. C., Sullivan, G.B. y Allen, F.C.L. (2008). A theoretical model of EGM problem gambling: More than a cognitive escape. International Journal of Mental Health and Addiction, DOI 10.1007/s11469-008-9152-6

Weatherly, J.N., Sauter, J.J. y King, B.M. (2004). The "big win" and resistance to extinction when gambling. Journal of Psychology, 138, 495-504.

Welte, J.W., Barnes, G.M, Wieczorek, W.F., Tidwell, M-C, O. y Hoffman, J.H. (2007). Type of gambling and availability as risk factors for problem gambling: A Tobit regression analysis by age and gender. International Gambling Studies, 7, 183-198. doi.org/10.1080/14459790701387543.

Welte, J.W., Wieczorek, W.F., Barnes, G.M., Tidwell, M.-C. y Hoffman, J.H. (2004). The relationship of ecological and geographic factors to gambling behavior and pathology. Journal of Gambling Studies, 20, 405-423. doi. org/10.1007/s10899-004-4582-y.

Williams, R.J. (2010). Pre-Commitment as a Strategy for Minimizing Gambling-Related Harm. Informe presentado al Parlamento de Australia. Julio 2010.

Williams, R.J. (2014). Prevention of Problem Gambling: Evidence and indentified best practice. Comunicación presentada al 3th International Symposium of Excessive Gambling, Neuchätel, Febrero de 2014.

Williams, R.J., West, B. y Simpson, R. (2012). Prevention of Problem Gambling. Report prepared for the Ontario Problem Gambling Research Centre and the Ontario Ministry of Health and Long Term Care. October 1, 2012. 